\title{
Se préparer efficacement aux défis de demain
}

\section{Bruno Kesseli, texte et photos}

Dr med. et lic. phil., rédacteur en chef

Le deuxième symposium MedEd, qui s'est tenu le 23 septembre 2015 à Berne, s'est penché sur les «Perspectives de la formation médicale». En réunissant un panel d'experts pour un échange approfondi sur les questions en lien avec la formation des médecins, cette rencontre a répondu aux attentes et permis d'esquisser les grandes lignes (de pensée) en vue de préparer ce domaine aux enjeux du système de santé de demain.

«Nous ne voulons pas être un simple centre administratif», a déclaré Werner Bauer, président de l'ISFM, dans son discours d'introduction du deuxième symposium MedEd. Il est certain que les tâches administratives constituent une part importante de la mission de l'ISFM. Le président a cependant souligné qu'il lui tenait à cœur de positionner l'ISFM comme un promoteur actif de la formation médicale. A cet égard, le deuxième symposium MedEd a été un succès. La rencontre a été ponctuée de plusieurs présentations remarquées, suscitant un débat approfondi et la poursuite des idées développées.

\section{Activités diversifiées de l'ISFM}

Werner Bauer a rappelé les objectifs que poursuit le symposium au-delà de l'échange général d'informations et d'idées: «Identification et analyse des enjeux et défis», «Discussion des différentes options pour organiser la formation postgraduée et continue», «Débat sur les nouveautés méthodologiques» et enfin "Ouverture vers l'étranger». Il a ensuite présenté une série de chiffres impressionnants soulignant la diversité des activités de son Institut. L'ISFM gère 10000 médecins-assistants et 1500 centres de formation postgraduée; il élabore, actualise et met en œuvre près de 100 programmes de

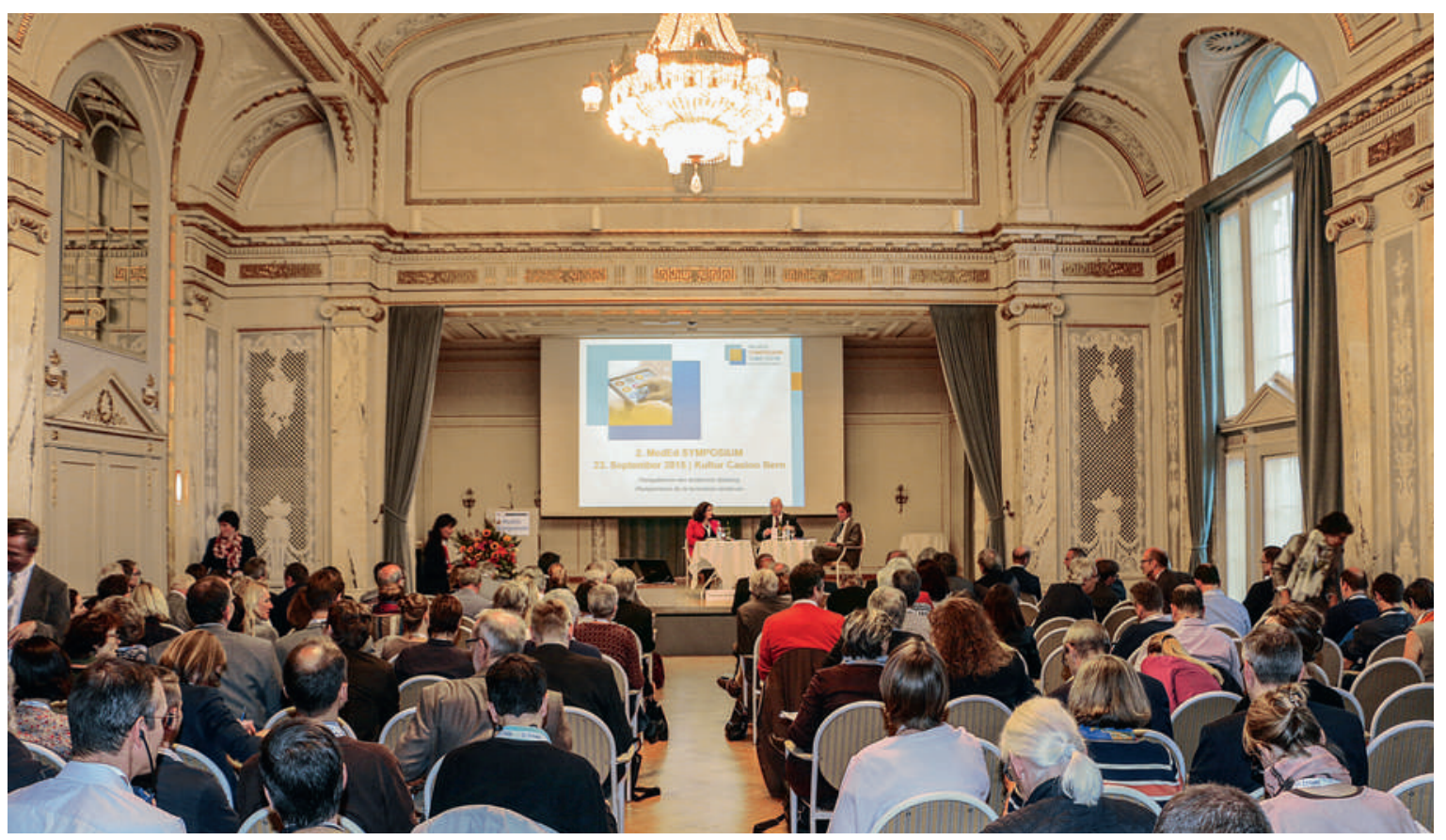

Cette année encore, le symposium MedEd s'est révélé être une rencontre captivante pour un public spécialisé. 
formation; il traite chaque année 2000 demandes de titre et environ 1000 demandes de renseignements de médecins étrangers; et enfin il organise près de 100 visites d'établissements, pour ne quantifier que quelquesunes de ses tâches. Le nombre de titres de spécialiste octroyés n'a cessé d'augmenter ces dernières années pour arriver à 1501 l'année dernière. $44 \%$ des titres sont délivrés à des médecins détenteurs d'un diplôme étranger, la plus grande partie provenant d'Allemagne (62\%) suivie de l'Autriche (12\%) et de l'Italie (7\%). Ces chiffres montrent que la formation dans notre pays est encore loin de pouvoir couvrir le besoin en médecins indigènes. Mais ce ne sont pas les seuls défis que Werner Bauer a identifié. Il a notamment aussi évoqué les changements structurels du domaine de la santé et de la société, l'importance du "teaching», le financement de la formation postgraduée, le champ de tension entre formation et services, les nouvelles pistes pour définir les objectifs de formation, des méthodes d'évaluation pertinentes ou la régulation de la formation et de l'admission des médecins.

\section{Coordonner au lieu de réguler}

Ce dernier point, sujet explosif de la politique de la santé, a été également repris par Pascal Strupler. Pour le directeur de l'Office fédéral de la santé publique, la solution au problème, selon lequel la répartition des médecins n'est pas toujours adaptée aux besoins, ne réside pas dans une régulation centralisée avec des quotas, qu'il considère de toute façon irréalisable en Suisse. «Nous devons trouver d'autres pistes», a-t-il déclaré en citant notamment les possibilités d'améliorer la coordination de la prise en charge et d'approfondir la collaboration interprofessionnelle. Il a également rappelé la situation insatisfaisante des données de la

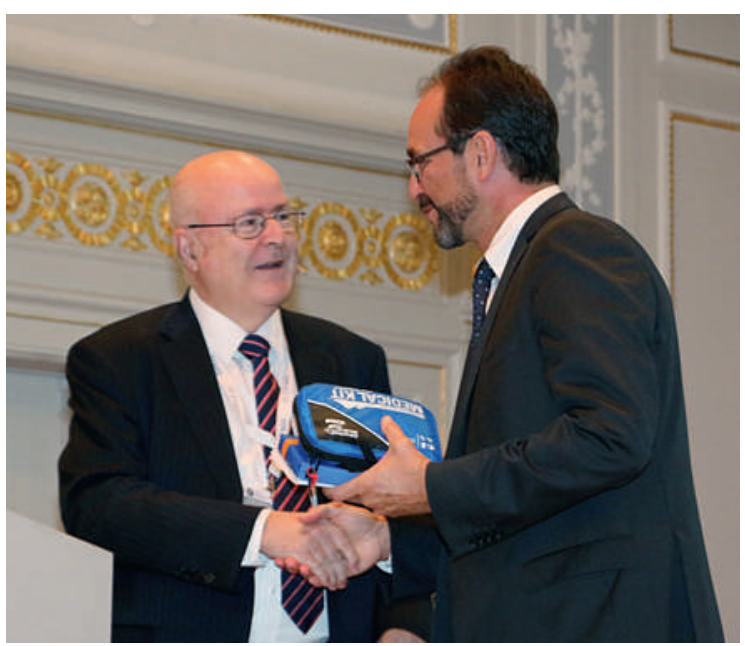

Les petits présents sont un témoignage de l'amitié: Werner Bauer (à gauche) remercie Pascal Strupler après son exposé.

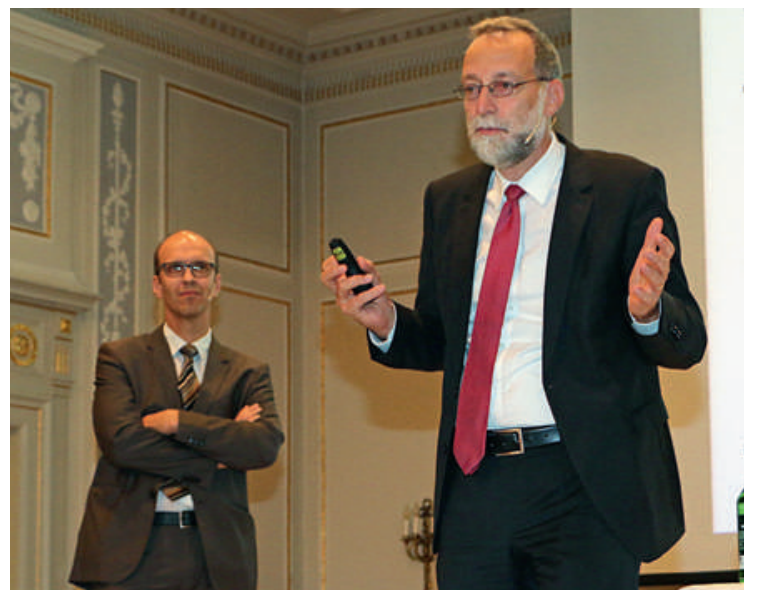

Interaction fructueuse: Rolf Zehnder (à droite) et Stefan Breitenstein évoquent la formation médicale postgraduée dans I'environnement hospitalier.

médecine ambulatoire et la volonté de l'OFSP «d'éclaircir ces zones d'ombre». Ces données sont en effet primordiales pour relever les défis à venir comme l'augmentation de l'espérance de vie à laquelle est liée la hausse des maladies chroniques, a-t-il encore ajouté en précisant avoir ressenti une certaine peur du corps médical face à trop de transparence.

S'agissant du manque de relève médicale formée en Suisse, Pascal Strupler se montre optimiste. Il rappelle la hausse déjà réalisée du nombre de places d'études et l'offre à venir à l'EPFZ, à Fribourg, St-Gall et au Tessin. Selon ses prévisions, à moyen terme, il ne sera plus question de pas assez mais de trop de places d'études. Le directeur de l'OFSP voit l'ISFM comme un intermédiaire entre les différents acteurs impliqués dans la formation médicale. Il partage l'avis de Werner Bauer selon lequel la collaboration entre l'OFSP et l'ISFM est bonne. Et dans la droite ligne de «Qui aime bien, châtie bien", lorsque Werner Bauer fait allusion au petit nombre de médecins à l'OFSP, il lui répond que, si c'est ainsi, c'est sans nul doute lié à l’attractivité du TARMED...

\section{Le duo dynamique de Winterthour}

Bien plus qu'un exemple concret, l'intervention de Rolf Zehnder et Stefan Breitenstein a donné une véritable leçon de ce que pourrait être une interaction fructueuse entre l'«administration" et la "pratique». Dans sa fonction de directeur de l'hôpital cantonal de Winterthour (HCW), R. Zehnder s'est penché sur la question de «La formation médicale: frein ou moteur du développement des hôpitaux»". En s'adjoignant le directeur du département Chirurgie de l'HCW, Stefan Breitenstein, afin de garantir un lien concret avec la pratique médicale, il a ajouté une dynamique supplémentaire à leur message. Leur exposé a été un plaidoyer convain- 
cant et convaincu visant à (continuer à) accorder beaucoup d'importance à la formation médicale en milieu hospitalier même si les conditions cadres sont en perpétuelle mutation.

Une des déclarations phare de leur présentation a été de dire que du point de vue stratégique, la formation des médecins est primordiale pour tout le domaine hospitalier mais aussi pour le positionnement des hôpitaux, en précisant que la création de réseaux avec les institutions de formation revêt une importance croissante. Ils ont résumé leur vision d'un «développement fructueux de la formation médicale dans les hôpitaux" en sept thèses aussi pertinentes que précises. "Nous voulons avoir l'obligation de former», clament-ils dans la "Thèse 1: la formation des médecins est du ressort des hôpitaux». Pendant trop longtemps, le secteur ne s'est pas assez soucié de la formation des médecins, ont-ils signalé dans un élan d'(auto)critique et, vu qu'au final, ce secteur les forme pour ses propres besoins, il doit aussi se sentir concerné et responsable de cette mission. Le côté structurel de la formation postgraduée d'aujourd'hui doit être abandonné au profit de modèles axés sur les compétences et, dans la foulée, il faut promouvoir de nouvelles professions comme celle de Clinical Nurse ou de Physician Assistant qui, indirectement, améliorent le quotidien professionnel des médecins en formation. Toujours selon eux, les meilleures conditions pour la formation postgraduée sont offertes par les réseaux et des structures entrepreneuriales. Dans leur modèle, l'ISFM aurait également un rôle décisif à jouer pour autant qu'il puisse agir en tant qu'institution forte et indépendante au sein d'une collaboration institutionnalisée avec les hôpitaux et les autres acteurs de la formation médicale.

\section{Etre un spécialiste borné est une question de caractère}

La question traitée par Johann Steurer dans son exposé a été la suivante: «Formation postgraduée: rénovation

\section{ISFM-Award pour un engagement exceptionnel \\ dans la formation postgraduée}

Werner Bauer a profité du deuxième symposium MedEd pour présenter brièvement les lauréats de I'ISFM-Award 2015 pour un engagement exceptionnel dans la formation postgraduée. Les formateurs suivants, nommés par leurs anciens médecins-assistants, ont été récompensés:

Beat Althaus, Zurich; Christian Begemann, Schaffhouse; Marco Conti, Lugano; Andreas Günthert, Lucerne; Martina Hafner, Bâle; Evelyn Hartmann-Mohr, Thoune; Lukas Hefermehl, Baden; Christian Kellenberger, Zurich; Christoph Kniestedt, Zurich; Andreas Kühne, Buchs; François Kundig, La Chaux-de-Fonds; Sébastien Martin, Lausanne; Thorsten Meuthen, Altstätten; Thi Dan-Linh Nguyen-Kim, Zurich; Sebastian Olbrich, Zurich; Olivier Pasche, Thierrens; François Scerba, Nyon; Reto Sutter, Zurich; Sebastian Walther, Berne; Susanne Wegener, Zurich; Rainer Wolf, Berne; Wolfgang Zaunbauer, St-Gall.

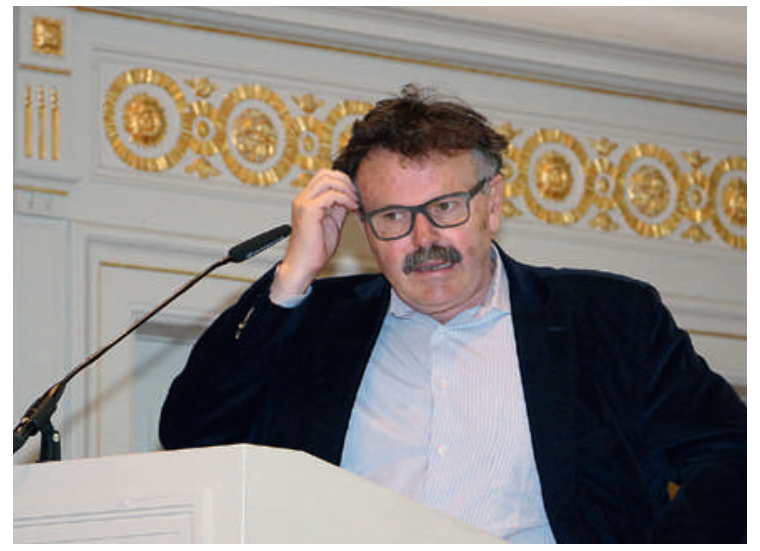

Reconstruction inévitable: Johann Steurer aimerait délester la formation prégraduée du superflu.

en douceur ou refonte totale?». Le directeur du Centre Horten pour la recherche orientée vers la pratique et le transfert de connaissances de l'Université de Zurich est nettement favorable à la deuxième méthode. Le principe d'une rénovation en douceur n'a pas fonctionné, déclare-t-il, si bien qu'il est inévitable de passer par une refonte totale. Le concept encore très répandu de la connaissance des données et des faits ne fonctionne pas, il est illusoire de croire qu'on puisse former des médecins susceptibles d'avoir une vue d'ensemble de toute la médecine. Aujourd'hui, un médecin devient spécialiste à 37 ans en moyenne. Cet objectif devrait être atteint selon lui à 30 ans au plus tard. Sa proposition de solution consiste à délester la formation prégraduée: la formation de base (niveau bachelor) doit servir à l'enseignement de ce que tous doivent savoir, «quel qu'en soit le contenu» - alors que la spécialisation commence dès le master. Afin de désamorcer les principaux contre-arguments, il les a anticipés. A ceux qui croient que la spécialisation commence trop tôt avec ce modèle, il répond que cette crainte n'est pas fondée. Les apprentis doivent déjà prendre de telles décisions dès l'âge de 15 ans. Il n'y voit pas non plus de risque d'encourager une vision unilatérale et renfermée de la spécialisation: «Etre un spécialiste borné est une question de caractère», conclut-il, et les candidats prédisposés ne manquent pas. A l'image des discussions pendant la pause, les déclarations de J. Steurer ont suscité le débat. Il sera intéressant d'observer les éventuels effets à long terme.

\section{Termes élogieux pour la Suisse romande}

Le ton a été très harmonieux lors de l'entretien mené ensuite par Werner Bauer avec Catherine Gasser et Michael Jordi sur le thème de la «Formation médicale le rôle de la Confédération et des cantons». Aussi bien la responsable de la division Professions de la santé de 


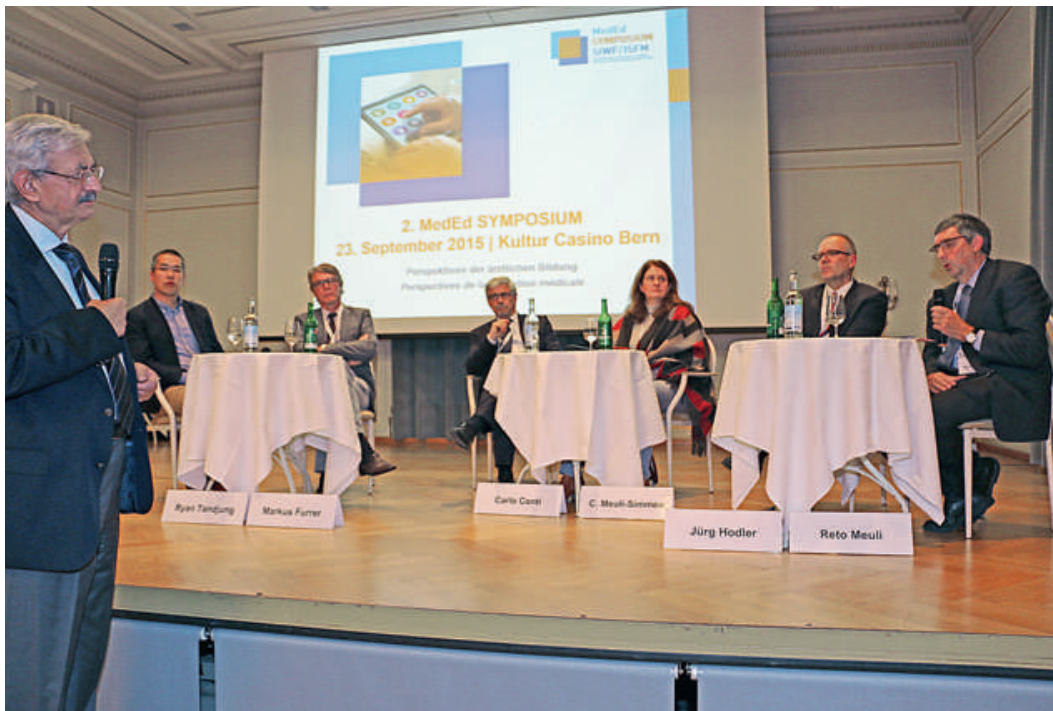

Des avis très pointus: Iwan Rickenbacher (à gauche) et les orateurs de la table ronde.

l'OFSP que le secrétaire central de la Conférence suisse des directrices et directeurs cantonaux de la santé (CDS) revendiquent un "fédéralisme coordonné», au centre duquel trône le dialogue entre les acteurs. En définitive, tous les partenaires sont liés pour le meilleur et pour le pire, estime M. Jordi qui inclut aussi l'ISFM et la FMH. Comme Pascal Strupler, Catherine Gasser souligne la bonne coopération avec l'ISFM alors qu'elle ressent des tensions importantes au sein de la FMH, entre généralistes et spécialistes, qui engendrent occasionnellement des réflexes de protection à l'image de l'équation «Berset = médecine étatique». Ce genre de simplifications ont le défaut de briser les chances de réussir en matière d'innovation et de bonnes solutions élaborées conjointement. En revanche, C. Gassler a été élogieuse envers la Suisse romande qui joue un rôle de pionnier par sa coopération offensive dans le domaine de la formation prégraduée, postgraduée et continue.

\section{Puisse le chemin rester cahoteux}

Sur son ton habituel, plein d'humour et d'esprit, le philosophe et journaliste Ludwig Hasler s'est penché sur la question du «chemin cahoteux entre objectif de formation et compétence». S'il a formulé l'espoir que le chemin reste cahoteux, il se pose aussi la question de savoir qu'est-ce que «l'homme juste». Il relève un trait de caractère qui lui semble essentiel chez l'être humain, son manque de clarté, son besoin de toujours se réajuster, ce qui, justement, lui confère une certaine «drôlerie». Les médecins ne devraient pas être les ministres d'une époque mais pouvoir accomplir leur vocation et tences élémentaires des médecins qui ne peuvent pas être transmises sous forme d'objectifs de formation. «Je parle là de ce qu'ils doivent être et ne peuvent pas obtenir simplement.» Pour la formation prégraduée, postgraduée et continue, L. Hasler plaide en faveur de «davantage de réflexion» et "davantage de confrontation", et il cite en exemple l'EPFZ qui entreprend beaucoup pour inciter le partenariat entre des systèmes de pensée hétérogènes. Il a conclu par une recommandation: ne croyez pas qu'apprendre c'est «accumuler des informations».

\section{Concepts de formation: plus de flexibilité par la pression économique?}

L'après-midi a commencé par quatre ateliers* parallèles sur les thèmes suivants:

- changement structurel du secteur de la santé et formation postgraduée;

- formulation des objectifs de formation, assessment, évaluation et recertification, aujourd'hui et demain;

- nouvelles méthodes didactiques: e-Learning, Blended Learning, Simulation;

- diagnostic pédagogique pour les internes/assistants en difficulté de raisonnement clinique.

Dans le dernier exposé de la journée, Martin Fischer s'est demandé à quoi pourrait ressembler «le développement des compétences médicales tout au long de la vie en période de pression économique». Le directeur de l'Institut de didactique et de recherche sur la formation en médecine de l'Université de Munich voit l'avenir dans la flexibilité des concepts de formation et dans une formation prégraduée et postgraduée basée sur les compétences. Cela présuppose cependant davantage de souplesse dans la manière de comprendre les rôles du formateur et de la personne en formation. Il estime que la pression économique n'a pas que des effets négatifs car la vision économique pourrait livrer des arguments pour davantage de flexibilité dans les concepts de formation.

«Avez-vous recommandé à votre fille de devenir médecin?» Telle est la question posée d'emblée par Iwan Rickenbacher aux invités de la table ronde "Les défis d'aujourd'hui et leurs conséquences sur la formation des médecins». Le professeur honoraire de communication politique de l'Université de Berne a réussi à obtenir des orateurs présents, Carlo Conti, Markus Furrer, Jürg Hodler, Claudia Meuli-Simmen, Reto Meuli et Ryan Tandjung, des avis très pointus sur les principaux thèmes de la journée et à finir ainsi en beauté cette rencontre passionnante pour un public très concerné par le sujet. 